\title{
Sistema de Secado Solar para Frutos Tropicales
}

\author{
Antonio R.S. Costa y Sebastiaõ R. Ferreira \\ Universidad Federal de Río Grande del Norte, Departamento de Ingeniería Química, \\ 59078-970 Natal, RN-Brasil (e-mail: ferreira@eq.ufrn.br, seba@ufrnet.br)
}

\begin{abstract}
Resumen
Se presenta la construcción de un secador solar en el que se realizaron ensayos de secado de frutos tropicales y se desarrolla el modelado matemático simplificado del proceso. Se empleó un sistema de adquisición de datos y control de temperatura de secador, usando energía de convección forzada de aire y radiación solar. El sistema es complementado, para emergencia, con resistencias eléctricas. Se realizaron experimentos de secado de bananas en forma de placa, obteniéndose sus pesos y contenidos acuosos en función del tiempo. Se realizó el modelado matemático del proceso de secado, con la ecuación de transferencia de materia, obteniéndose la difusividad del agua en los frutos. Los valores obtenidos concuerdan con datos de la literatura y pueden ser empleados en el diseño preliminar del secador. El sistema estudiado entrega información para calcular la cantidad de agua evaporada del alimento para una determinada temperatura promedio del secador solar, la potencia y el tiempo necesarios para el secado.
\end{abstract}

Palabras clave: secado solar, frutos tropicales, energía solar, difusión de agua

\section{Drying Solar System for Tropical Fruits}

\begin{abstract}
The construction of a solar dryer is presented in this work. Experimental runs for the drying of tropical fruits were performed and a simplified model of the drying process. A data acquisition system was used and the drying temperature was controlled by means of an air stream heated by a simultaneous process of forced convection and solar radiation. The system also included electric resistances, for emergency situations. Drying experiments were performed with banana slabs, monitoring their weight and water content as a function of time. A simplified mathematical model was developed considering only the mass transfer equation, estimating the water diffusivity on the fruits. The values of these diffusivities are in agreement with other values reported in the literature, so they can be used in a preliminary design of solar dryer. The system studied provides a way of calculating the amount of water to be evaporated in the dryer at a particular mean temperature, the work power to be supplied and the required drying time.
\end{abstract}

Keywords: solar drying, tropical fruits, solar energy, diffusion of water 


\section{INTRODUCCIÓN}

El secado es uno de los procesos comerciales más usados en la conservación de productos agropecuarios, sin que ellos pierdan mucho sus propiedades organolépticas y nutricionales, y por ser un proceso económico si comparado a otros que emplean temperaturas próximas a la temperatura ambiente. El proceso de secado de un alimento consiste en la remoción de parte de su agua libre hasta llegar a un nivel tal que él pueda ser consumido y/o almacenado en un lugar a temperatura ambiente.

Algunos métodos empleados para realizar el secado son:

a) Secado natural, en el cual, dependiendo del tipo de producto, él es disperso, por ejemplo, en terrenos cementados, de ladrillos o en la planta de secado.

b) Secado artificial, es realizado en secadores mecánicos, en los que se hace circular aire caliente, impulsado por ventiladores, sobre el producto a ser secado. El aire puede ser calentado con gas de la combustión de leña, de la quema de combustibles fósiles o aún por electricidad.

Con la crisis energética y el aumento de los precios de los combustibles, han aumentado considerablemente los costos del secado por métodos convencionales, tal como el secado mecánico, exigiendo la búsqueda de otras metodologías para realizar el secado. Un procedimiento de secado, que combina el secado mecánico con el natural, es usado en Brasil principalmente para granos. El mismo consiste en combinar la energía colectada como radiación solar y convección forzada del aire, realizada por ventiladores, resultando en el aprovechamiento tanto de la radiación solar así como de la convección forzada.

Comúnmente, en ese sistema de secado se necesita solamente un complemento de energía eléctrica para mover el ventilador que impulsa el aire. Cuando se obtiene un producto seco por este método, por ejemplo, banana pasa, el valor de comercialización del producto en Brasil es aproximadamente 10 veces el valor de compra del alimento in natura, lo que asegura buena ganancia a los inversionistas.

Para modelar rigurosamente un sistema de secado se necesita que las ecuaciones de balance de materia y energía sean resueltas simultáneamente, en el interior del alimento. Sin embargo, una simplificación importante se obtiene al adimensionalizar las ecuaciones de Fourier (energía) y Fick (materia), y analizar la razón entre la difusividad molecular $D_{A B}\left(\mathrm{~m}^{2} / \mathrm{s}\right)$ y térmica $\alpha\left(\mathrm{m}^{2} / \mathrm{s}\right)$. Para frutos cortados en rodajas de pequeño espesor, cuando $D_{A B} \ll \alpha$, es decir, la resistencia a la difusión de materia es mucho mayor que la resistencia a la difusión de calor, el secado puede modelarse considerando únicamente el balance de materia (ecuación de Fick).

En la presente investigación fue construido y realizados ensayos en un secador solar para frutos tropicales, con un sistema de adquisición de datos y control de temperatura de secado. Se realizaron ensayos de secado obteniéndose datos de peso del alimento y de su contenido acuoso en función del tiempo. Fue modelado de forma simplificada el secado, usando sólo la ecuación de transferencia de materia, para calcular la difusividad molecular del agua en los frutos.

\section{SECADOR ELÉCTRICO-SOLAR}

El sistema de secado, presentado en la Fig.1, fue desarrollado para el trabajo doctoral de uno de los autores de este artículo (Antônio. R. S. Costa) y para la maestría de otro investigador (Dejanilton F. Rêgo), el cual diseñó un sistema supervisor y de control del secador eléctrico-solar. Básicamente, en el esquema del aparato de secado hay un colector solar, una cámara de secado, un extractor eólico y un ventilador acoplado a un motor para promover la convección forzada del aire caliente.

En la Fig. 1, el aire impulsado por el ventilador se mueve en el colector calentándose, pasando, después, en el compartimiento de resistencias eléctricas, que aportan energía, cuando es necesario complementar la energía solar, para obtener la temperatura escogida de secado. Entonces, el aire entra en la cámara de secado, en la cual el alimento es secado debido al movimiento del aire caliente, además del aprovechamiento de la energía solar que entra directamente por las paredes de la cámara, ya que ellas son hechas con un polímero transparente. El aire que es succionado por el extractor eólico, sale para el 
ambiente. El colector solar tiene $5 \mathrm{~m}$ de largo y 0,8 $\mathrm{m}$ de diámetro, fue hecho con polímero transparente, flexible y su forma es similar a la de un cigarrillo encendido. El colector es liviano si es comparado con colectores clásicamente usados en secado solar; que son generalmente pesados, difíciles de transportar y a veces no removibles.

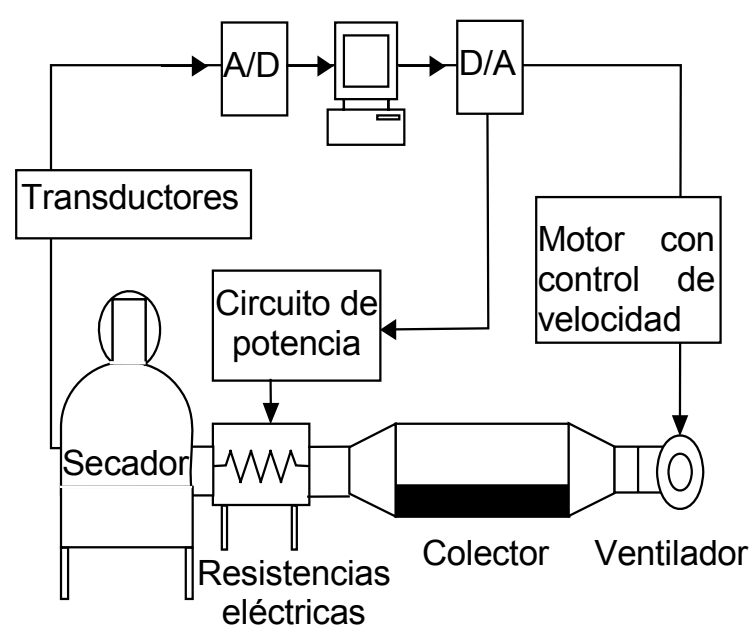

Fig. 1. Sistema de secado con energía solar, convección forzada y resistencias eléctricas

Fue realizada la automatización del aparato de secado. El control de temperatura de secado es realizado por un circuito de potencia eléctrica, con el cual es posible complementar la energía para el secado, cuando la energía solar no es suficiente para obtenerse la temperatura de procesamiento del alimento.

Los valores de variables del proceso, como temperatura, que es colectada con termopares, son grabados en un microcomputador, en el cual está instalada una placa de adquisición de datos. Un sistema supervisor fue desarrollado para visualizar y para procesar las variables medidas. El programa supervisor y de control en tiempo real fue desarrollado en lenguaje de programación a través del programa LabVIEW-5.1 de la empresa National Instruments.

\section{MODELOS DE SECADO}

Para la obtención de modelos de secado se usan las ecuaciones básicas de fenómenos de transporte, o sea, la ecuación de transferencia de materia, energía y cantidad de movimiento. Cuando son adimensionalizadas las ecuaciones de transporte o las condiciones de contorno, surgen los números adimensionales como el número de Biot, Fourier y Luikov; los cuales son definidos en este artículo. En esos números adimensionales están incluidos parámetros que influyen en el secado, como la difusividad molecular del agua en el fruto $D_{A B}\left(\mathrm{~m}^{2} / \mathrm{s}\right)$ y el coeficiente convectivo de transferencia de calor $\mathrm{h}\left(\mathrm{W} \mathrm{m}^{-2}{ }^{\circ} \mathrm{C}^{-}\right.$ $\left.{ }^{1}\right)$.

Existen investigaciones en las que se dedican al secado de banana, como en la tesis de Lima (1999), reproducidas parcialmente por Lima et al. (2002). Él desarrolló modelos matemáticos bidimensionales, analíticos y numéricos para simular el fenómeno de difusión en sólidos y, en particular, ellos fueron usados para describir la transferencia de materia y energía durante el secado de banana. Fue usada una condición de contorno convectiva o, entonces, la de equilibrio en la superficie del sólido, además, él empleó el coeficiente de difusión constante o aun variable y contempló la posibilidad de la existencia de encogimiento del alimento en el secado.

Lima (1999), estudió varias situaciones como, por ejemplo, modificándose los números de Biot (Bi) y Fourier (Fo). Obtuvo ecuaciones para las difusividades $\alpha$ y $D_{A B}$, el coeficiente convectivo $h$ y el coeficiente externo de transferencia de materia $\mathrm{k}_{\mathrm{M}}(\mathrm{m} / \mathrm{s})$, empleando el método de los mínimos cuadrados, para el cálculos de los parámetros. Los números de Biot y Fourier de transferencia de calor y materia son 
definidos, respectivamente, como:

$$
\begin{aligned}
& \mathrm{Bi}_{C}=\frac{h L}{k} \\
& \mathrm{Bi}_{M}=\frac{k_{M} L}{D_{A B}} \\
& F_{C}=\frac{\alpha t}{L^{2}}=\frac{k t}{\rho C p L^{2}} \\
& F_{M}=\frac{D_{A B} t}{L^{2}}
\end{aligned}
$$

Siendo $\mathrm{Cp}\left(\mathrm{Jkg}^{-10} \mathrm{C}^{-1}\right)$ el calor específico del objeto (fruto), $\mathrm{k}\left(\mathrm{Wm}^{-10} \mathrm{C}^{-1}\right)$ su conductividad térmica, $\mathrm{L}(\mathrm{m}) \mathrm{su}$ dimensión característica y $\rho\left(\mathrm{kgm}^{-3}\right)$ su densidad. El número de Biot de transferencia de calor, $\mathrm{Bi}_{\mathrm{C}}=\mathrm{hL} / \mathrm{k}=$ $(1 / \mathrm{k} / \mathrm{L}) /(1 / \mathrm{h})$, es la relación entre la resistencia interna a la transferencia de calor, $(1 / \mathrm{k} / \mathrm{L})$ y la resistencia a la transferencia de energía por convección, $1 / \mathrm{h}$. El número de Biot de transferencia de materia, $\mathrm{Bi}_{\mathrm{M}}=$ $\left(1 / D_{A B} / L\right) /\left(1 / k_{M}\right)$, es la relación entre la resistencia interna a la transferencia de materia, $1 / D_{A B} / L$, y la resistencia externa, $1 / \mathrm{k}_{\mathrm{M}}$. Cuanto mayor es la difusividad $\alpha=k /(\rho C p) \circ D_{A B}$, para parámetros finitos $\mathrm{ty} L$, más rápido ocurre la difusión de calor o de materia en el objeto bajo análisis y más rápido se logra llegar en él, a la temperatura o a la concentración escogida.

Cuando los números de Biot, $\mathrm{Bi}_{\mathrm{C}}$ y $\mathrm{Bi}_{\mathrm{M}}$, están en los rangos $0,1<\mathrm{Bi}_{\mathrm{C}}<100$ y $0,1<\mathrm{Bi}_{\mathrm{M}}<100$, existen tanto la resistencia interna como la externa a la transferencia de calor y materia, respectivamente. Cuando los números de Biot $\mathrm{Bi}_{C}<0,1 \mathrm{y} \mathrm{Bi}_{\mathrm{M}}<0,1$ no existen resistencias internas y cuando $\mathrm{Bi}_{C}>100$ y $\mathrm{Bi}_{\mathrm{M}}>100$ no existen resistencias externas (Luikov, 1968). En algunos libros, como el de Heldman y Singh (1981), en vez del límite 100 se considera el valor 40 para el límite de los dos números de Biot.

Cuando una fruta está cortada en láminas de pequeño espesor, se puede considerar que existe sólo transferencia de materia, pues el material queda casi isotérmico en pocos minutos. Es decir, en el inicio del secado hay aporte de energía para calentar el alimento a través del calor sensible hasta que él llegue a una temperatura casi constante. A partir de este instante, la energía es suministrada sólo para evaporar agua, a través del calor latente y la ecuación de conducción de energía ya no tiene importancia en el interior del sólido.

Numerosos modelos tienen sido propuestos para describir la temperatura, la concentración acuosa de productos agrícolas así como su velocidad de pérdida de humedad durante el secado, tales como los difusivos, empíricos y semiempíricos, además de los basados en la termodinámica de los procesos irreversibles.

Los modelos difusivos generalmente están basados en la difusión de líquido y/o vapor dentro del producto. Con ellos son obtenidos los perfiles de temperatura y de concentración de agua, en función de la posición adentro del sólido y del tiempo.

Los modelos empíricos y semiempíricos en general son empleados para describir el secado de una partícula o de una capa fina o delgada de partículas. Estos modelos son, casi siempre, obtenidos de simplificaciones de las soluciones de las ecuaciones diferenciales de los modelos difusivos. Segun Parti (1993), los modelos simplificados, sin resistencia interna, a veces llamados lumped models, son 
aplicables a la transferencia de calor y materia, cuando los números de Biot valen, respectivamente, $\mathrm{Bi}_{\mathrm{C}}$ $<1,5$ y $\mathrm{Bi}_{\mathrm{M}}<10$. En esos rangos de números de Biot, aún son importantes tanto la resistencia interna como la externa a la transferencia de energía y materia, pero aun así ellas fueron parcialmente despreciadas por Parti (1993), para obtener modelos para capa delgada.

Uno de los modelos más conocidos es el de Luikov (1966), basado en la termodinámica de los procesos irreversibles (Abalone et al., 2001; Luikov, 1966; Pandey et al., 1999; Wu y Irudayaraj, 1996). En el modelo propuesto por Luikov (1966) el proceso es descrito por un conjunto de ecuaciones diferenciales parciales acopladas para la temperatura, humedad y en casos de intenso secado, también, puede incluir la presión.

En los últimos años fueron realizadas varias investigaciones en las que son contemplados además del modelado de secado de frutos (Lima, 1999), también el modelo del secador, como en las investigaciones de Karim y Hawlader (2005a, b) y de Mabrouk et al. (2006). Esos dos modelos están relacionados, pues la masa de agua perdida por el alimento es transferida para el aire usado en el secado, que pertenece al llamado modelo de equipo. Los dos modelos se conectan y se acoplan a través de los flujos de materia y energía en la interfase fruta/aire.

Karim y Hawlader (2005a, b) desarrollaron un modelo de transferencia de materia y energía, para el secado convectivo de frutos tropicales. El modelo del material (banana) es capaz de predecir temperatura y humedad instantáneas adentro del fruto. Es admitido el encogimiento del material y, debido a esto, el coeficiente de difusión no es constante, pero es dependiente del contenido acuoso y de la temperatura del fruto (Bird et al., 2002, Crank, 1976). También fueron obtenidas ecuaciones para el secador, denominado modelo del equipo, las cuales son usadas para predecir los cambios que ocurren en el equipo durante el proceso de secado.

Es decir, predicen la temperatura y la humedad instantáneas del aire de secado a lo largo del secador tipo túnel. Ellos presentaron datos de coeficientes de difusión de agua en bananas a temperaturas de 40 , 50 y $60^{\circ} \mathrm{C}$ y velocidades del aire de secado de $0,3,0,5$ y $0,7 \mathrm{~m} / \mathrm{s}$. Según ellos, la velocidad de secado es mucho más dependiente de la temperatura que de la velocidad del aire.

Este resultado se debe a que la resistencia externa no cambia mucho o es despreciable, en el rango usado de velocidad del aire de secado. La difusividad obtenida por ellos disminuye con el progreso del proceso de secado; lo mismo fue observado en los cálculos realizados a partir de los datos de la presente investigación; pero fueron presentados sólo los valores promedios de difusividad. Es decir, debido al encogimiento son producidos cambios en la estructura microscópica del fruto y, por esto, aumenta la resistencia a la difusión del agua, disminuyendo su coeficiente de difusión.

Karim y Hawlader (2005a, b) concluyeron que, con los modelos desarrollados por ellos, se obtienen buenas predicciones de la velocidad de secado, perfiles de temperatura y humedad en bananas y de perfiles de temperatura y humedad a lo largo del secador. Compararon datos experimentales obtenidos en un secador solar y valores calculados, usando rodajas de banana y concluyeron que ellos concuerdan bien; prediciendo temperatura y humedad en el secador tipo túnel, así como en las rodajas de banana durante su secado. Mabrouk et al. (2006) desarrollaron un modelo numérico para la predicción de la transferencia de materia y energía de productos granulares en un secador tipo túnel, de lecho fijo. Como la capa usada de producto (uva) era de pequeño espesor, admitieron que no existía gradiente de temperatura y humedad en la capa de material, y obtuvieron un modelo simplificado basado en una capa delgada. Emplearon, por ejemplo, una capa de frutos, admitidos como placas, de $0,8 \mathrm{~cm}$ de espesor, velocidades del aire de secado de 0,5 a $7 \mathrm{~m} / \mathrm{s}$ y temperatura de entrada de $60{ }^{\circ} \mathrm{C}$, en un túnel de $6 \mathrm{~m}$.

Después de conducir una serie de experimentos, en los rangos de temperatura de entrada de aire de 40 a $80^{\circ} \mathrm{C}$, velocidad del aire de 1 a $7 \mathrm{~m} / \mathrm{s}$ y humedades relativas de 5 a $50 \%$, Mabrouk et al. (2006) concluyeron que la temperatura y velocidad del aire influyen mucho en la velocidad de secado. Según ellos, el régimen de flujo del aire es turbulento $\mathrm{y}$, en consecuencia, la superficie del material, directamente expuesta al secado, se acerca rápidamente a la humedad de equilibrio, pero los cambios en el interior de la capa de material es lenta. 


\section{MODELO PROPUESTO DE SECADO}

Se presenta un modelo simplificado de secado, basado en el análisis de parámetros, a partir de la segunda ecuación de Fourier de transferencia de energía y la segunda de Fick de transferencia de materia.

En general el flujo de humedad debido a gradientes de presión no es significativo para temperaturas convencionales de secado, pero solamente a temperaturas mucho mayores que esas. Por lo tanto, comúnmente los términos de presión pueden ser eliminados y las ecuaciones resultantes son:

$$
\begin{aligned}
& \frac{\partial \mathrm{u}_{\mathrm{A}}}{\partial \mathrm{t}}=\nabla^{2}\left(\mathrm{~K}_{11} \mathrm{u}_{\mathrm{A}}\right)+\nabla^{2}\left(\mathrm{~K}_{12} \mathrm{~T}\right) \\
& \frac{\partial \mathrm{T}}{\partial \mathrm{t}}=\nabla^{2}\left(\mathrm{~K}_{21} \mathrm{u}_{\mathrm{A}}\right)+\nabla^{2}\left(\mathrm{~K}_{22} \mathrm{~T}\right)
\end{aligned}
$$

Siendo $\mathrm{u}_{\mathrm{A}}$ ( $\mathrm{kg}$ de agua/kg de sólido) la humedad, $\mathrm{T}\left({ }^{\circ} \mathrm{C}\right)$ la temperatura, $\mathrm{t}(\mathrm{s})$ el tiempo, $\mathrm{Kij}, \mathrm{i}, \mathrm{j}=1$ y 2 , son los coeficientes fenomenológicos para $\mathrm{i}=\mathrm{j}$ y los coeficientes combinados para $\mathrm{i} \neq \mathrm{j}$.

Los efectos de gradientes de temperatura en la difusión de humedad sólo resultan significantes en el secado por conducción o cuando hay un intenso calentamiento, tal como ocurre en el secado por microondas o secado dieléctrico. Debido a estas consideraciones, las ecuaciones fenomenológicas, se transforman en:

$$
\begin{aligned}
& \frac{\partial \mathrm{u}_{\mathrm{A}}}{\partial \mathrm{t}}=\nabla^{2}\left(\mathrm{~K}_{11} \mathrm{U}_{\mathrm{A}}\right) \\
& \frac{\partial \mathrm{T}}{\partial \mathrm{t}}=\nabla^{2}\left(\mathrm{~K}_{22} \mathrm{~T}\right)
\end{aligned}
$$

Estas ecuaciones serían las obtenidas a partir del modelo de Luikov, después de admitidas las consideraciones y simplificaciones realizadas aquí. Es decir, las ecuaciones resultantes son sólo las difusivas, en las que los coeficientes fenomenológicos $K_{11}$ e $K_{22}$ son la difusividad de materia (agua) $D_{A B}$ y la difusividad térmica $\alpha$, respectivamente.

Sea, entonces, una placa de espesor $2 \mathrm{~L}(\mathrm{~m})$, constituida por rodaja de fruta (banana), a través de la cual hay flujo unidimensional y simétrico de energía y materia en ambas superficies, es decir, en $x=-L$ y $x=+$ L. Dividiendo la Ec.(7) por la (8) y considerando como constantes $K_{11}=D_{A B}$ y $K_{22}=\alpha=k /(\rho C p)$, resulta de la adimensionalización de citadas ecuaciones:

$\frac{D_{A B}}{\alpha}=\frac{1 / \alpha}{1 / D_{A B}}=L u=\frac{1}{L e}=\frac{\frac{\partial^{2} T}{\partial x^{2}} /(\partial T / \partial t)}{\frac{\partial^{2} u_{A}}{\partial x^{2}} /\left(\partial u_{A} / \partial t\right)}$

El número de Luikov es representado por $L u=(1 / \alpha) /\left(1 / D_{A B}\right)=($ resistencia interna a la difusión de calor)/(resistencia interna a la difusión de materia). Si el número de Luikov, es $L u<<1$, significa que la transferencia de materia domina el proceso simultáneo de transferencia de calor y materia, pues ella seria una etapa mucho más lenta que la de transferencia de calor. El inverso del número de Luikov es conocido como número de Lewis, Le. Con las consideraciones hechas y admitiendo que no hay 
resistencia externa a la transferencia de energía, la Ec.(7) es la ecuación diferencial básica para el análisis, usando las condiciones de contorno presentadas a seguir:

$$
\begin{aligned}
& u_{A}(x ; 0)=u_{A 0} \quad t=0 \\
& -D_{A B} \rho_{S} \frac{d u_{A}}{d x}=0 \quad x=0 \\
& -D_{A B} \rho_{S} \frac{d u_{A}}{d x}=h_{M}\left(u_{A s}-u_{A \infty}\right) \quad x=L
\end{aligned}
$$

La Ec.(10) representa la condición inicial, la Ec.(11) la de simetría, en $x=0$ y la Ec.(12) el flujo de materia en la superficie de la placa, en $x=L$. En la Ec.(12) el parámetro $u_{A S}$ es la concentración acuosa de equilibrio justamente en la superficie del alimento, en $x=L, u_{A \infty}$ es la concentración acuosa en el medio externo lejos de la superficie, $h_{M}\left(k m^{-2} s^{-1}\right)$ un parámetro, $k_{M}=h_{M} / \rho_{s}$ el coeficiente externo de transferencia de materia y $\rho_{s}\left(\mathrm{~kg} / \mathrm{m}^{3}\right)$ la densidad de los sólidos en el alimento. En la Ec.(12), a veces, es conveniente usar la presión parcial próxima a la superficie, $\mathrm{p}_{\mathrm{AS}}$, y lejos de ella, $\mathrm{p}_{\mathrm{A} \infty}$, en vez de las concentraciones $u_{A S} y u_{A \infty}$, respectivamente. A través de la adimensionalización de la condición de contorno convectiva, dada por la Ec.(12), se obtiene el número de Biot, $B i_{M}=\left(h_{M} / \rho_{S}\right) L / D_{A B}=k_{M} L / D_{A B}$, de la Ec.(2).

Cuando el número de Biot, $B \mathrm{~B}_{M}=\left(\mathrm{h}_{\mathrm{M}} / \rho_{\mathrm{S}}\right) \mathrm{L} / \mathrm{D}_{\mathrm{AB}} \rightarrow \infty$, es decir, para el límite $\mathrm{Bi}_{\mathrm{M}}>100$, la condición de contorno en $\mathrm{x}=\mathrm{L}$, dada por la Ec.(12), se transforma en una concentración constante $\mathrm{u}_{\mathrm{A}}=\mathrm{u}_{\mathrm{AS}} \rightarrow \mathrm{u}_{\mathrm{A} \infty}$, es decir:

$\mathrm{u}_{\mathrm{A}}=\mathrm{u}_{\mathrm{As}}=\mathrm{u}_{\mathrm{A} \infty} \quad \mathrm{x}=\mathrm{L}$

La solución de la Ec.(7), con las condiciones de contorno dadas por las Ec.(10), (11) y (13), suministra la humedad puntual de agua, o sea, la masa de agua/masa de sólido en el alimento, en función del tiempo y de la posición, $u_{A}(x ; t)$. Integrando esa solución para todo el volumen de la placa, incluyendo su espesor, de $x=-L a x=+L$, se obtiene la humedad promedio, es decir, la masa promedio de agua/masa de sólido, en función del tiempo, $\bigcup_{A}(\mathrm{~kg}$ agua $/ \mathrm{kg}$ de sólido $)=U_{\mathrm{A}}(\mathrm{t})$. Cuando el número de Biot, $\mathrm{Bi}_{\mathrm{M}} \rightarrow \infty$, es decir, para el límite $\mathrm{Bi}_{\mathrm{M}}>100$, la solución obtenida (Crank, 1976), en términos de humedad promedio $U_{A}$, es:

$\frac{U_{A}-U_{A \infty}}{U_{A 0}-U_{A \infty}}=\sum_{n=0}^{\infty} \frac{8}{\pi^{2}(2 n+1)^{2}} \exp \left\{\frac{-D_{A B} \pi^{2}(2 n+1)^{2} t}{4 L^{2}}\right\}$

En la Ec.(14) $U_{A 0}$ y $U_{A \infty}$ es, respectivamente, la humedad promedio en el alimento en el tiempo cero e infinito. Para cálculos de ingeniería, para Fourier $\mathrm{Fo}_{\mathrm{M}}=\mathrm{D}_{\mathrm{AB}} \mathrm{t} / \mathrm{L}>0,15$ es suficiente usar solamente el primer término de la serie de la Ec.(14), con una desviación menor que $\mid 1,5 \%$ en el cálculo del tiempo de secado.

La difusividad puede ser obtenida de la Ec.(14) y es útil para el diseño preliminar del secador. La humedad perdida por el alimento puede ser calculada, a una dada temperatura promedio, a través de la derivada de la Ec.(14) y su posterior integración desde el tiempo $t=0$ hasta el tiempo escogido $t=t$, obteniéndose para una rodaja de banana que $\Delta U_{A}=U_{A 0}-U_{A t}$. Se puede evaluar la potencia $(\mathrm{J} / \mathrm{s})$ necesaria para el funcionamiento de un secador solar, multiplicando la entalpía de vaporización del agua $\Delta \mathrm{H}_{\mathrm{VAP}}(\mathrm{J} / \mathrm{kg})$, por la masa de agua evaporada de todas las $(\mathrm{n})$ rodajas de frutos $(\mathrm{n}) \Delta \mathrm{U}_{\mathrm{A}} \mathrm{M}_{\mathrm{S}}(\mathrm{kg})$ y dividiendo por el tiempo del proceso $t(\mathrm{~s})$; resultando en la potencia $(\mathrm{J} / \mathrm{s})=\Delta \mathrm{H}_{\mathrm{VAP}}(\mathrm{n})\left(\mathrm{U}_{\mathrm{A} 0}-\mathrm{U}_{\mathrm{At}}\right) \mathrm{M}_{\mathrm{S}} / \mathrm{t}$; siendo $\mathrm{M}_{S}(\mathrm{~kg}) \mathrm{la}$ 
masa total de sólidos en una rodaja de fruto. A esta energía puede ser sumada la suministrada al alimento como calor sensible, para cambiar su temperatura desde la temperatura inicial hasta la final promedio de secado.

Además, la difusividad puede ser empleada en el cálculo preliminar del tiempo de secado, $\mathrm{t}(\mathrm{s})$, usándose la Ec.(14), a través de un método simplificado de cálculo. Ese tiempo puede ser obtenido, a una temperatura promedio elegida y difusividad correspondiente, de la derivada de la Ec.(14) y su posterior integración desde la humedad inicial $U_{A 0}$ al tiempo $t=0$, hasta la humedad $U_{A}$, que corresponde al tiempo para el secado $t(s)$.

Cuando las resistencias externas tanto debido a la transferencia de materia como de energía son despreciables o pequeñas en relación a las transferencias internas, resulta útil analizar el proceso solamente con las resistencias difusivas internas, como fue discutido en el modelado de esta investigación. Sablani et al. (2000) llegaron a conclusiones similares a las presentadas aquí. Ellos admitieron que la solución de la segunda ecuación de Fick puede ser empleada, sin la segunda de Fourier, cuando el encogimiento es despreciable o no es considerado, la resistencia a la difusión de materia es mayor en el interior del alimento (no hay resistencia externa), son despreciables los efectos internos y externos de transferencia de calor, y son despreciables los efectos transientes iniciales.

\section{RESULTADOS Y DISCUSIÓN}

Son presentados, en la Tabla 1, algunos datos experimentales de secado de varias rodajas de banana, en el mencionado secador solar, en la cual están la masa total de agua $M_{A}(g)$ y de sólidos $M_{S}(g)$. Empleando la Ec.(14), a varias temperaturas del aire de secado $T_{A}$, fueron calculadas difusividades del agua en banana, $D_{A B}$, que son presentadas en citada tabla.

Lima (1999), consideró en particular, el límite para el coeficiente $k_{M}$ tendiendo a infinito, $B i_{M}=k_{M} L / D_{A B} \rightarrow$ $\infty$, admitiendo los parámetros $L$ y $D_{A B}$ finitos; el cual resulta en una situación límite de análisis, similar a la desarrollada en el presente trabajo. Los valores calculados por Lima (1999), para difusividad de agua en bananas, a temperaturas similares a las de la Tabla 1, son: para $T_{A}=39,9^{\circ} \mathrm{C}, D_{A B}=1,4010^{-10} \mathrm{~m}^{2} / \mathrm{s}$, para $\mathrm{T}_{\mathrm{A}}=49,9^{\circ} \mathrm{C}, \mathrm{D}_{\mathrm{AB}}=2,3110^{-10} \mathrm{~m}^{2} / \mathrm{s}$ y para $\mathrm{T}_{\mathrm{A}}=60,2^{\circ} \mathrm{C}, \mathrm{D}_{\mathrm{AB}}=3,0810^{-10} \mathrm{~m}^{2} / \mathrm{s}$. Las difusividades calculadas por la Ec.(14) y presentadas en la Tabla 1 , son del mismo orden de magnitud de los valores evaluados por Lima (1999). Karim y Hawlader (2005 a,b) obtuvieron difusividades de agua en banana, por ejemplo, para velocidad del aire de secado de $0,7 \mathrm{~m} / \mathrm{s}$ : para $T_{A}=40^{\circ} \mathrm{C}, D_{A B}=0,9810^{-10} \mathrm{~m}^{2} / \mathrm{s}$, para $T_{A}=50^{\circ} \mathrm{C}, D_{A B}$ $=1,7410^{-10} \mathrm{~m}^{2} / \mathrm{s}$ y para $\mathrm{T}_{\mathrm{A}}=60^{\circ} \mathrm{C}, \mathrm{D}_{\mathrm{AB}}=2,4110^{-10} \mathrm{~m}^{2} / \mathrm{s}$.

Tabla 1: Datos experimentales para rodajas de banana con espesor promedio inicial de $1 \mathrm{~cm}$ y difusividades calculadas en este trabajo

\begin{tabular}{|l|l|l|l|l|l|}
\hline \multicolumn{2}{|l|}{$\mathrm{T}=40^{\circ} \mathrm{C}$} & \multicolumn{2}{l|}{$\mathrm{T}=50{ }^{\circ} \mathrm{C}$} & \multicolumn{2}{l|}{$\mathrm{T}=60{ }^{\circ} \mathrm{C}$} \\
$\mathrm{M}_{\mathrm{S}}=64,5 \mathrm{~g}$ & \multicolumn{2}{l|}{$\mathrm{M}_{\mathrm{S}}=77,6 \mathrm{~g}$} & \multicolumn{2}{l|}{$\mathrm{M}_{\mathrm{S}}=36,7 \mathrm{~g}$} \\
$\mathrm{D}_{\mathrm{AB}}\left(\mathrm{m}^{2} / \mathrm{s}\right)=$ & \multicolumn{2}{l|}{$\mathrm{D}_{\mathrm{AB}}\left(\mathrm{m}^{2} / \mathrm{s}\right)=$} & \multicolumn{2}{l|}{$\mathrm{D}_{\mathrm{AB}}\left(\mathrm{m}^{2} / \mathrm{s}\right)=$} \\
$2,12.10^{-10}$ & \multicolumn{2}{l|}{$2,44.10^{-10}$} & \multicolumn{2}{l|}{$2,75.10^{-10}$} \\
\hline $\mathrm{t}(\mathrm{h})$ & $\mathrm{M}_{\mathrm{A}}(\mathrm{g})$ & $\mathrm{t}(\mathrm{h})$ & $\mathrm{M}_{\mathrm{A}}(\mathrm{g})$ & $\mathrm{t}(\mathrm{h})$ & $\mathrm{M}_{\mathrm{A}}(\mathrm{g})$ \\
\hline 0,5 & 150,8 & 0,5 & 168,6 & 0,5 & 130,2 \\
\hline 1,0 & 144,5 & 1,0 & 159,8 & 1,0 & 101,4 \\
\hline 1,5 & 139,5 & 1,5 & 151,3 & 1,5 & 84,1 \\
\hline 2,0 & 135,7 & 2,0 & 144,8 & 2,0 & 72,5 \\
\hline 2,5 & 132,6 & 2,5 & 138,8 & 2,5 & 62,9 \\
\hline 3,0 & 129,6 & 3,0 & 133,9 & 3,0 & 53,9 \\
\hline 3,5 & 124,4 & 3,5 & 126,6 & 3,5 & 41,7 \\
\hline 4,0 & 120,2 & 4,0 & 118,5 & 4,0 & 31,1 \\
\hline 5,0 & 116,2 & 5,0 & 112,3 & 5,0 & 22,6 \\
\hline 6,0 & 113,0 & 6,0 & 107,1 & 6,0 & 15,0 \\
\hline 7,0 & 109,9 & 7,0 & 102,6 & 7,0 & 6,6 \\
\hline--- & --- & 8,0 & 98,4 & 8,0 & 3,5 \\
\hline
\end{tabular}


Para temperatura de $27^{\circ} \mathrm{C}$ Lima (1999), calculó la difusividad térmica de banana como siendo $\alpha=$ $\mathrm{k} /(\rho \mathrm{Cp})=1,4710^{-7} \mathrm{~m}^{2} / \mathrm{s}$, usando los datos $\mathrm{k}=0,481 \mathrm{Wm}^{-10} \mathrm{C}^{-1}, \rho=980 \mathrm{~kg} / \mathrm{m}^{3}$ y Cp $=3.346 \mathrm{Jkg}^{-10} \mathrm{C}^{-1}$. Empleando esa difusividad térmica en el cálculo preliminar para temperatura $\mathrm{T}_{\mathrm{A}}=39,9^{\circ} \mathrm{C}$, despreciando cambios de las propiedades con la temperatura, y usando $D_{A B}=1,4010^{-10} \mathrm{~m}^{2} / \mathrm{s}$, evaluada por Lima (1999), se obtiene el número de Luikov, $L u=0,000952=1 / \mathrm{Le}=1.050=\left(1,4010^{-10} \mathrm{~m}^{2} / \mathrm{s}\right) /\left(1,4710^{-7} \mathrm{~m}^{2} / \mathrm{s}\right)$. Por lo tanto, el proceso de secado en el interior del alimento, para este análisis realizado por Lima (1999), está dominado por la difusión interna de materia, porque el número de Luikov Lu $<<1$.

Para la temperatura $T_{A}=40^{\circ} \mathrm{C}$ se obtiene, de la Tabla 1 , la difusividad molecular $D_{A B}=2,1210^{-10} \mathrm{~m}^{2} / \mathrm{s}$ y usando la difusividad térmica $\alpha=1,47.10^{-7} \mathrm{~m}^{2} / \mathrm{s}$, obteniendo un número de Luikov Lu $=\left(2,1210^{-10}\right.$ $\left.\mathrm{m}^{2} / \mathrm{s}\right) /\left(1,4710^{-7} \mathrm{~m}^{2} / \mathrm{s}\right)=0,00144=1 / \mathrm{Le}=693,4$. Lo que confirma el predominio de la difusión interna de materia en relación a la difusión interna de calor, para el experimento analizado, al emplear el modelo desarrollado en la presente investigación, es decir, cuando el número de Biot tiende a infinito $\left(\mathrm{Bi}_{\mathrm{M}} \rightarrow \infty\right)$.

\section{CONCLUSIONES}

Fue presentado un modelo para el cálculo de la difusividad de agua en alimentos, a partir de consideraciones exclusivamente difusivas de transferencia de materia y energía en el interior de frutos, basándose en las soluciones de las ecuaciones de Fick y Fourier, respectivamente. Resultando, por lo tanto, que el cálculo de la difusividad puede ser realizado empleando solamente la solución de la ecuación de Fick, porque la difusión de materia es mucho más lenta que la difusión de calor en el interior del fruto.

Los valores de difusividades obtenidas, a partir del modelo analítico simplificado de la presente investigación, concuerdan con las difusividades evaluadas a través de un método numérico empleado por Lima (1999); presentado mejor precisión para $\mathrm{T}_{\mathrm{A}}=50$ y $60^{\circ} \mathrm{C}$. Los valores de difusividad calculados por Karim y Hawlader (2005a, b), a temperaturas $T_{A}=50$ y $60{ }^{\circ} \mathrm{C}$, son del mismo orden de magnitud de los evaluados en la presente investigación. Los datos de difusividad pueden ser empleados en el diseño preliminar de un secador, partiendo del cálculo de la masa agua evaporada de todas las $(n)$ rodajas de los frutos $(n)\left(U_{A 0}-U_{A t}\right) M_{S}(\mathrm{~kg})$, multiplicando por de la entalpía de vaporización del agua $\Delta H_{V A P}(J / k g)$ y dividiendo por el tiempo t(s) para el secado, resultando en la potencia mínima para el secado, dada por $\Delta \mathrm{H}_{\mathrm{VAP}}(\mathrm{n})\left(\mathrm{U}_{\mathrm{AO}}-\mathrm{U}_{\mathrm{At}}\right) \mathrm{M}_{\mathrm{S}} / \mathrm{t}$.

\section{REFERENCIAS}

Abalone, R., A. Gastón y M.A. Lara, Simulación Numérica del Proceso de Secado de un Material Anisotrópico, Engenharia Térmica: $\mathrm{n}$ ํ 1, 47-55 (2001).

Bird, R.B., W.E. Stewart y E.N. Lightfoot, Transport Phenomena, John Wiley, New York, USA (2002).

Crank, J., The Mathematics of Diffusion, Clarendon Press, Oxford, England (1976).

Heldman, R.H. y R.P. Singh, Food Process Engineering, AVI Publishing Company, Westport, Connecticut (1981).

Karim, Md.A. y M.N.A. Hawlader, Drying Characteristics of Banana: Theoretical Modelling and Experimental Validation, Journal of Food Engineering, 70, 35-45 (2005a).

Karim, Md.A. y M.N.A. Hawlader, Mathematical Modelling and Experimental Investigation of Tropical Fruits Drying, International Journal of Heat and Mass Transfer, 48, 4914-4925 (2005b).

Lima, A.G.B., Fenómeno de Difusión en Sólidos Esferoidales Prolatos. Estudio de Caso: Secado de Banana, Tesis, UNICamp - Universidad Estadual de Campinas, Facultad de Ingeniería Mecánica, Campinas, Brasil (1999). (En portugués.) 
Lima, A.G.B., M.R. Queiroz y S.A. Nebra, Simultaneous Moisture Transport and Shrinkage During Drying of Solids with Ellipsoidal Configuration, Chemical Engineering Journal, v.86, 85-93 (2002).

Luikov, A.V, Analytical Heat Diffusion Theory. Academic Press, New York, USA (1968).

Luikov, A.V., Heat and Mass Transfer in Capillary Porous Bodies, Pergamon Press, New York, USA (1966).

Mabrouk, S.B., B. Khiari y M. Sassi, Modelling of Heat and Mass Transfer in a Tunnel Dryer, Applied Thermal Engineering, 26, 2110-2118 (2006).

Pandey, R.N., S.K. Srivastava y M.D. Mikhailov, Solutions of Luikov Equations of Heat and Mass Transfer in Capillary Porous Bodies Through Matrix Calculus: a New Approach, International Journal of Heat and Mass Transfer, 42, 2649-2660 (1999).

Parti, M., Selection of Mathematical Models for Drying Grain in Thin-layers, Journal of Agricultural Engineering Research, 54, 339-352 (1993).

Sablani, S., S. Rahman y N. Al-habsi, Moisture Diffusivity in Foods - an Overview, In A. S. Mujumdar (Ed.), Drying Technology in Agriculture and Food Sciences, pp 35-50, Enfield, Science Publishers Inc (2000).

Wu, Y. y J. Irudayaraj, Analysis of Heat, Mass and Pressure Transfer in Starch Based Food Systems, Journal of Food Engineering, 29, 399-414 (1996). 